\title{
Expression profiling, gene silencing and transcriptional networking of metzincin metalloproteases in the cattle tick, Rhipicephalus (Boophilus) microplus
}

Annette-Christi Barnard ${ }^{a}$, Ard M. Nijhof ${ }^{\text {b,c }}$, Anabella R. M. Gaspar ${ }^{a}$, Albert W. H. Neitz ${ }^{a}$, Frans Jongejan ${ }^{b, d}$, Christine Maritz-Olivier ${ }^{\mathrm{e}}$

a Department of Biochemistry, Faculty of Natural and Agricultural Sciences, University of Pretoria, Pretoria, 0002, South Africa.

b Utrecht Centre for Tick-borne Diseases (UCTD), Department of Infectious Diseases and Immunology, Faculty of Veterinary Medicine, Utrecht University, Yalelaan 1, 3584CL Utrecht, The Netherlands.

c Institute for Parasitology and Tropical Veterinary Medicine, Freie Universität Berlin, Königsweg 67, 14163 Berlin, Germany.

d Department of Veterinary Tropical Diseases, Faculty of Veterinary Science, University of Pretoria, Private Bag X04, 0110 Onderstepoort, South Africa.

e Department of Genetics, Faculty of Natural and Agricultural Sciences University of Pretoria, Pretoria, 0002 , South Africa

Corresponding author: Maritz-Olivier, C. (christine.maritz@up.ac.za). Phone: +27 124203945 / +27 12 420 3258. Fax: +27 123625327 / +27 123625302 


\section{Abstract}

Tick proteins functioning in vital physiological processes such as blood meal uptake, digestion and reproduction are potential targets for anti-tick vaccines, since vaccination could inhibit these essential functions and ultimately affect tick survival. In this study we identified metzincin metalloproteases from Rhipicephalus microplus as potential vaccine candidates since they are implicated as essential to blood-cavity formation, bloodmeal digestion and reproduction in ixodid ticks. Eight transcripts encoding proteins that contain the characteristic metzincin zinc-binding motif HEXXHXXG/NXXH/D and a unique methionine containing "methionine-turn" were identified from native and in-house assembled R. microplus expressed eequence tag (EST) databases. These were representative of five reprolysin-like and three astacin-like metzincin metalloproteases. Reverse transcription-PCR analysis indicated that the reprolysins were most abundantly expressed in the salivary glands, whereas the astacins were most abundant in the midgut and ovaries. In vivo gene silencing was performed to assess a possible phenotype of these metalloproteases during adult female $R$. microplus blood feeding and reproduction. RNA interference (RNAi) against two of the reprolysins and one of the astacins significantly affected the average egg weight and oviposition rate. Evidently, this reverse genetic approach enabled the evaluation of the overall vital impact of tick proteins. Finally, integrated real time-PCR studies also revealed an extensive cross organ network between the R. microplus metzincin transcripts, supporting the use of a combinatorial metzincinbased anti- $R$. microplus vaccine. 
Keywords Metzincin metalloproteases, Rhipicephalus microplus, Expression profiling, In vivo $\mathrm{RNAi}$

\section{Introduction}

The impact of ticks as obligate haematophagous ectoparasites and disease vectors on livestock productivity is immense (Jongejan and Uilenberg, 2004). Rhipicephalus (Boophilus) microplus is endemic in most subtropical and tropical regions of the world, causing annual economic losses in the hundreds of millions of dollars to cattle producers (Jongejan and Uilenberg, 2004; Estrada-Pena et al., 2006). A rising concern is the continued expansion of its distribution range, especially in areas where it is accompanied by the displacement of Rhipichephalus (B.) decoloratus (Macleod, 1978; Mason and Norval, 1980; Tonnesen et al., 2004). While $R$. decoloratus only efficiently transmits Babesia bigemina, R. microplus is a competent vector of both B. bigemina and Babesia bovis. Since the latter protozoan is more pathogenic and results in much heavier losses in susceptible livestock, the concern regarding $R$. microplus displacing $R$. decolorates is obvious. Besides the pathogens transmitted by $R$. microplus such as Anaplasma marginale, Babesia spp. and Borrelia theileri, heavy tick loads also cause direct harm to hosts as their feeding behavior results in blood loss, scar tissue formation and leather damage. Currently the control of $R$. microplus relies primarily on chemical acaricides. However, apart from being a pollutant to the environment, these substances augment acaricide-resistant $R$. microplus populations (Baffi et al., 2007). Considering all of the above, it is clear that it is of great importance to create an alternative and more effective control strategy, to counter the negative economical impacts. 
One of the most attractive alternative control approaches is to exploit the host's immunity through vaccination of livestock against ticks (Willadsen, 2004). Vaccination of livestock offers the advantages of being cost-effective, reducing environmental contamination and preventing the selection of acaricide-resistant ticks. Furthermore, by reducing tick populations and/or affecting tick vector capacity, vaccines can also indirectly prevent or reduce transmission of pathogens (de la Fuente et al., 1998; , 2007). The most limiting step for the improvement and/or development of new vaccines, however, remains the identification and characterization of effective vaccine antigen targets (Jongejan et al., 2007).

Based on various studies, metzincin metalloproteases have been recognized as key bioactive components in vital physiological processes of invertebrates, and therefore are considered as promising targets for control strategies against medical and economical important micro-organisms and parasites. These enzymes are multi-domain endopeptidases, which are distinguished by an extended zinc-binding consensus motif (HEXXHXXG/NXXH/D, where $X$ represent any amino acid) in the active site and a conserved methionine-containing turn (Met-turn) underlying the active site (Bode et al., 1993; Gomis-Rüth, 2009). To date, seven metzincin families have been analyzed at the structural level, including the astacin and reprolysin family (reviewed by Gomis-Rüth, 2009).

Through protein degradation, protein - protein interaction modulation, extra-cellular matrix (ECM) turn-over and highly specific cleavage events, these enzymes are involved in a diverse range of essential physiological processes. Vector-borne protozoa are known to require these metalloproteases for replication and use them as virulence factors (Barrett et 
al., 2004; Gomis-Rüth, 2003.) Parasites such as heamatophagous ectoparasites find metzincins essential for reproduction (Bowles et al., 2007; Young et al., 2000) and host infestation (Dzik, 2005; Gallego et al., 2005; Francischetti et al., 2003). These vital physiological implications of metzincins make them potentially attractive targets for vaccines.

Metalloproteases that form part of the array of known bioactive molecules in tick saliva have been classified as reprolysin-like metalloproteases based on sequence alignment analysis (Francischetti et al., 2003). To date six reprolysin-like metalloprotease coding sequences have been identified in Haemaphysalis longicornis (Harnnoi et al., 2007), five in Ixodes ricinus (Decrem et al., 2007; , 2008) and three in Ixodes scapularis (Francischetti et al., 2003). Based on sequence similarities, between the salivary gland metalloproteases and the haemorrhagic snake venom metalloproteases (SVMPs), and preliminary activity assays (Francischetti et al., 2003) it appears that these metalloproteases contribute to the facilitation and maintenance of a fluid blood feeding cavity during long extended feeding, by performing anti-haemostatic activities such as fibrinogenolysis and fibrinolysis. Accordingly, Decrem et al. (2007) proposed that these salivary gland metalloproteases are adequate vaccine candidates, since an elicited antibody response would result in the inhibition of essential anti - heamostatic enzymes (Decrem et al., 2007). Subsequent vaccination against these salivary gland metalloproteases did in fact affect the engorgement weight and fecundity of $I$. ricinus (with $47 \%$ and $55.4 \%$, respectively) (Decrem et al., 2007) and the mortality of $H$. longicornis (with 14.6\%) (Imamura et al., 2009). These reports support the notion that tick metzincin metalloproteases are promising anti-tick vaccine candidates and require further investigation to fully explore their potential. 
To date, metalloproteases have not been described in any ixodid tick tissue other than the salivary glands. However, various invertebrate studies have indicated metzincin metalloproteases, including reprolysins and astacins, in a wider variety of tissues with extensive roles in pathogen transmission (Barrett et al., 2004; Gomis-Rüth, 2003), host infestation (Williamson et al., 2006) and reproduction (Bowles et al., 2007; Freije and Bischoff, 2006). It is therefore anticipated that similar metzincins may also occur in a wider variety of tick tissues, with similar extensive roles, contributing to the tick's successful haematophagous parasitic behavior and its success as vector of disease.

Here we report the identification of reprolysin- and astacin-metzincin homologues from $R$. microplus, their expression profiles, phenotype assessment following in vivo RNAi, and evidence of a transcriptional interaction between these metzincins. This study provides a systematic approach to advance the use of metzincin metalloproteases as effective anti- $R$. microplus vaccines.

\section{Materials and methods}

\subsection{Experimental animals}

All animal experiments were performed at the Utrecht Centre for Tick-borne Disease (UCTD). Two 5-month-old male Holstein-Friesian calves (\#7766 and \#3903), which had no previous exposure to ticks, were used for tick feeding, during RNAi experiments. All tick feedings were approved by the Animal Experiments Committee (DEC) of the Faculty of Veterinary Medicine, Utrecht University (DEC No. 2008.II.07.068). 


\subsection{Ticks and tick feeding}

R. microplus ticks (Mozambique strain) were provided by ClinVet International (Pty), Bloemfontein, South Africa and were subsequently bred and maintained at the Utrecht Centre for Tick-borne Disease (UCTD), University of Utrecht. For tick feeding, patches with inner dimensions of $60 \mathrm{~mm} \times 85 \mathrm{~mm}$ were utilized. Five patches were glued to the shaved back of calf \#3903 and two patches to calf \#7766. Two batches of larvae (obtained from $1500 \mathrm{mg}$ pooled eggs, oviposited by 25 females) were placed in 2 different patches on day 0 (patch 1) and day 1 (patch 2) on calf \#7766. Approximately 400 unfed females were collected on day 16 and 200 unfed males were collected on day 16 and day 17 , respectively. The collected ticks were incubated at $27^{\circ} \mathrm{C}$ with $95 \%$ relative humidity. On day 16 the freshly molted females were injected with prepared dsRNA or injection buffer as described below.

\subsection{R. microplus reprolysin and astacin homologue identification}

Basic local alignment and search tool (BLAST) searches (using the blastp and PSI-blast algorithms) were performed against the $R$. microplus nucleotide sequences available at the National Centre for Biotechnology Information (NCBI) (http//:ncbi.nlm.nih.gov), the Bmi Gene Index (BmiGl) of expressed sequence tags (ESTs) available at the DFCI (http://compbio.dfci.harvard.edu/tgi/) and an in-house assembled $R$. microplus contig database. The amino acid sequence of Astacin (GenBank ID: CAA64981.1), the family prototype from Astacus astacus, and the I. scapularis reprolysin, MP1 (GenBank ID: AAP22067) (Francischetti et al., 2003) were used as queries, respectively. For sequence analysis, alignments were constructed using the ClustalX program (Thompson et al., 1997). 
2.4. Phylogenetic, topology and localization analysis

For phylogenetic analysis of the identified $R$. microplus astacin and reprolysin homologues, three available ixodid tick reprolysin-like MPs (I. scapularis MP1: GenBank

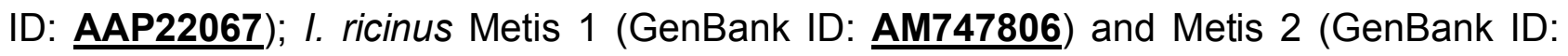

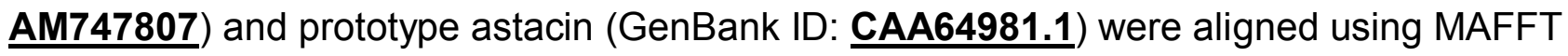
(v 6). Subsequent phylogenetic analysis was performed with the molecular evolution genetics analysis program (MEGA) (v 4.0) (Tamura et al., 2007) uitlizing neighbor-joining analysis (1000 bootstraps, substitution model: PAM Dayhoff matrix).

For localization predictions, theoretical signal peptides were determined utilizing the SignalP ( analysis was performed using the TMHMM server (v 2.0) (http://www.cbs.dtu.dk/services/TMHMM-2.0/).

\subsection{Expression profiling}

The expression profile of each transcript within eight female tissues (haemolymph, Malpighian tubules, ovaries, fat body of fed ticks and the midgut and salivary glands of both fed and unfed ticks), seven male tissues (testis of fed ticks and the accessory glands, midgut and salivary glands of both fed and unfed ticks) and over 10 lifestages (eggs, unfed larvae, engorged larvae, unfed nymphs, engorged nymphs, unfed female, unfed male, partially fed females, fed males and females after they oviposited all eggs) was determine using reverse transcriptional (RT)-PCR. Tissue and lifestage collection procedures and RNA isolation are described elsewhere (Nijhof et al., 2009; 2010). Firststrand cDNA was synthesized from $1 \mu \mathrm{g}$ total RNA using the first-strand cDNA synthesis system (Invitrogen ${ }^{\mathrm{TM}}$ ) and an oligo-dT primer. PCR amplification was performed using 
GoTaq (Promega, USA). PCR conditions were 35 cycles of 30 s at $94^{\circ} \mathrm{C}, 30$ s at the $\mathrm{Tm}$ of the gene specific primers and 30 s at $72^{\circ} \mathrm{C}$, preceded by an initial 2 min denaturation at $95^{\circ} \mathrm{C}$ and followed by a final extension at $72^{\circ} \mathrm{C}$ for $7 \mathrm{~min}$. PCR reactions were analyzed using agarose electrophoresis.

\section{6. dsRNA synthesis}

For each R. microplus metzincin transcript PCR-amplified cDNA templates were synthesized, utilizing gene specific primers, each with a T7 promoter sequence appended to the 5 ' end. Single stranded cDNA, synthesized from $R$. microplus fed mixed lifestages total RNA, served as initial template. All oligonucleotide primers used for dsRNA synthesis were synthesized by Invitrogen ${ }^{\mathrm{TM}}$ (Table 1). The region of each transcript's nucleic acid sequence which was targeted for silencing, were randomly selected and the sizes ranged from 120 - 635 bp. T7-incorporated PCR products were purified using the High Pure PCR Cleanup Micro Kit ${ }^{\mathrm{TM}}$ (Roche Diagnostics), and were used as templates to produce dsRNA using the T7 Ribomax Express RNAi system (Promega).

\subsection{Injection of ticks with dsRNA and phenotype analysis}

Injection of $R$. microplus females with dsRNA was performed as previously described in Nijhof et al. (2007). Freshly molted female ticks were injected with $0.5 \mu l$ dsRNA solution $\left(1-2 \times 10^{12}\right.$ molecules/ $\left.\mu \mathrm{l}\right)$ dissolved in injection buffer $(10 \mathrm{mM}$ Tris- $\mathrm{HCl}, \mathrm{pH} 7$ and $1 \mathrm{mM}$ EDTA) in the following combinations: BmMP4 (gi|71726986) and BmMP5 (gi|7172684) (Group A); BmMP1 (gi|71726988), BmMP2 (gi|71726992) and BmMP3 (gi|71726990) (Group B); As51 (gi|82845051) and As70 (gi|49558770) (Group C) and AsContig (assembled from gi|8285219, gi|82845107 and gi|82843217) (Group D). Each group comprised 80 ticks. A control group (Group TE), which was injected with injection buffer 
alone, was also included. Following injection the ticks were allowed to recover (for 2-5 hours at $27^{\circ} \mathrm{C}$ with $95 \%$ relative humidity), mortality due to injection was determined and each group (together with an excess of non-injected males) was placed in a separate patch on calf \#03903. Ticks were monitored daily. Female ticks that detached upon repletion were collected, weighed and placed at $27^{\circ} \mathrm{C}$ with $95 \%$ relative humidity for oviposition. Several parameters including: egg weight; percentage female weight converted into egg weight; and the hatching rate were monitored and compared between dsRNA-injected and control ticks. Statistical analysis was performed using one-way analysis of variance (ANOVA) and the Tukey-Kramer multiple comparison test with GraphPad InStat 3.10. Results were compared between the dsRNA and mock-injected ticks and $\mathrm{P}$ values of 0.05 or less were considered statistically significant.

\subsection{Gene silencing confirmation by semi-quantitative real-time PCR}

From each injected group, 15 partially fed females (with an average weight of $40 \mathrm{mg}$ ) were collected and dissected (giving three biological repeats, $n=5$ ). The salivary glands, midgut and ovaries were collected and total RNA was isolated from the collected tissues utilizing TRIzol (Invitrogen ${ }^{\mathrm{TM}}$ and Chomczynski, 1993). All samples were subsequently treated with DNase (Fermentas, Germany) and purified using both phenol/chloroform/isoamyl alcohol extraction and sodium acetate precipitation. cDNA was synthesized from 500 ng purified total RNA using the iScript ${ }^{\mathrm{TM}} \mathrm{CDNA}$ Synthesis Kit (BioRad Labrotories, Inc., USA). The synthesized cDNA was diluted 1/10 times with water and served as template.

The effect of RNAi on the transcription level of each transcript was analyzed by semiquantitative real-time PCR (semi-qPCR) using gene specific oligonucleotide primers 
designed to amplify a $\pm 100 \mathrm{bp}$ part, outside of the region that was targeted by dsRNA. All oligonucleotide primers used for real-time PCR were synthesized by Inqaba Biotec (Pretoria, South Africa) (Table 1). All real-time PCRs were performed in $10 \mu \mathrm{l}$ reaction volumes using the $\mathrm{KAPA}^{\mathrm{TM}}$ Syber $^{\circledR}$ FAST qPCR kit (KAPABiosystems, USA), on the LightCycler $^{\circledR} 480$ (Roche Diagnostics, Germany). For each transcript a standard curve was constructed, using a dilution series of $R$. microplus mixed lifestages as template. For each primer set a reaction lacking template was included as negative control. Run-to-run differences were corrected for by an intra-calibrator being incorporated in each run. For each reaction, three technical repeats were performed. Amplification consisted of a initial activation step at $95^{\circ} \mathrm{C}$ for 5 minutes followed by 45 cycles of $3 \mathrm{~s}$ at $95^{\circ} \mathrm{C}, 7 \mathrm{~s}$ at $55^{\circ} \mathrm{C}$ and 4s at $72^{\circ} \mathrm{C}$. Melting curve analysis, included 30 s at $95^{\circ} \mathrm{C}, 30 \mathrm{~s}$ at $55^{\circ} \mathrm{C}$, followed by a continuous acquisition mode from $95^{\circ} \mathrm{C}$, with a ramp rate of $0.1^{\circ} \mathrm{C} / \mathrm{s}$. Each reaction's $\mathrm{Cq}-$ value and melting temperature (Tm of the product) were calculated with the LightCycler $^{\circledR}$ 480 software and the data were analyzed with qbase ${ }^{\text {PLUS }}$ software (version 1.2) (Hellemans et al., 2007). All data were normalized against two reference genes, elongation factor 1-alpha (ELF1a) (GenBank ID: EW679365) and cyclophilin (PPIA)

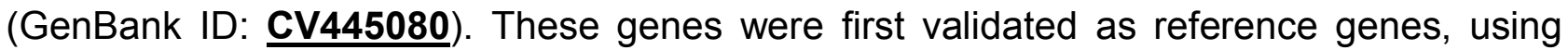
qbase $^{\text {PLUS }}$ : their respective coefficient of variance $(C V)$ among all tissues tested had to be less than $25 \%$ as per the acceptable limits of variation (Hellemans et al., 2007). To test the significance of each transcript's percentage silencing, an unpaired t-test with unequal variances was performed. The results were compared between the dsRNA and mockinjected tissues and P-values of 0.05 or less were considered statistically significant.

To assess if non-specific silencing occurred and to investigate the differential transcriptional responses between the transcripts, the expression level of each transcript 
within all the different dsRNA-injected groups (in all the relevant tissue types) was determined, using integrated semi-qPCRs. All reactions were conducted exactly as described above. Crude (non-normalized) Cq-data were exported to qbase ${ }^{\text {PLUS }}$, normalized to the two reference genes and statistically analyzed as described previously.

\section{Results}

\subsection{R. microplus astacin- and reprolysin-like homologue identification}

For reprolysin homologue identification, $R$. microplus EST sequences with the highest similarity $\left(\mathrm{E}\right.$-values $\left.\leq x \mathrm{e}^{-10}\right)$ to I. scapularis MP1 were obtained and aligned. This resulted in the identification of five complete coding sequence (cds) translating to five putative $R$. microplus reprolysins, namely: BmMP1 (gi|71726992), BmMP2 (gi|71726990), BmMP3 (gi|71726988), BmMP4 (gi|71726986) and BmMP5 (gi|7172684). The five reprolysin-like R. microplus homologues were aligned with I. scapularis MP1, I. ricinus Metis-1 and two reprolysin SVMPs (Fig 1).

It is evident that all five $R$. microplus sequences contain the typical zinc-binding (catalytic) domain of the reprolysin family (HEBGHXL/FGXXHD), except for one conserve substitution in BmMP1, BmMP2, BmMP3 and BmMP5, where the first glycine was substituted with an alanine. In BmMP4 the third residue of the motif is substituted with a cysteine, instead of the usual hydrophobic amino acid. The alignment against known reprolysins also indicates the conserved methionine adjacent to the catalytic domain (Fig 1). However, for the ixodid tick reprolysin-like sequences a unique conserved Met-turn motif is observed: GXXMSY. None of the R. microplus sequences contain the conserved 
RGD triplet typical of the disintegrin-domains present in some reprolysins. However, each of the five $R$. microplus reprolysin-like MPs possesses a cysteine-rich C-terminal domain, which is hypothesized to provide binding sites to extracellular matrix proteins (Iba et al., 1999).

For astacin homologue identification, the $R$. microplus EST sequences with the highest similarity $\left(E-v a l u e s \leq x \mathrm{e}^{-10}\right)$ to prototype astacin were identified and aligned. Three possible astacin-like R. microplus homologues were identified, namely: As51 (gil 82845051), As70 (gi|49558770) and AsContig (assembled from gi|82852194, gi|82845107 and gi|82843217) (Fig 2). All three putative tick astacin MPs contain the typical zincbinding (catalytic) domain of the astacin family (HEBXHA/VBGFXHE), except for one conserved glutamic acid to methionine substitution in As70. The conserved Met-turn is present in all three $R$. microplus astacin-like MPs, although the histidine directly adjacent to the methionine is substituted with a lysine, the structurally important tyrosine remains conserved. The typical astacin connecting segment (RXDRD), between the His-motif and the Met-turn is also present in all three $R$. microplus sequences.

3.2. Phylogenetic, topology and localization analysis of R. microplus reprolysin and astacin homologues

Phylogenetic analysis revealed the distinct evolutionary patterns of the two different metzincin families. Except for BmMP5, which branches independently, it is evident that the reprolysin R. microplus MPs, are closely related (Fig 3). As expected, the astacins grouped independently of the reprolysins (Fig 3). 
All five full length $R$. microplus reprolysin-like sequences contained a secretory signal peptide, indicating that these proteases are most likely secreted and act extracellularly (results not shown). Topology predictions were in agreement with these findings, since no transmembrane $\alpha$-helices were identified for any of the reprolysin sequences. For the three $R$. microplus astacin-like MPs the full-length coding sequences need to be determined. Therefore it can only be speculated that these $R$. microplus astacins (like all other invertebrate astacins) are not membrane associated, but most likely secreted (Sterchi et al., 2008).

\subsection{Expression profiling in R. microplus lifestages and tissues}

From the expression profiles it is evident that the reprolysin-like transcripts are most abundantly expressed in the salivary glands and that the astacins most abundantly in both female and male midgut and gonads (Fig 4). Both As51 and AsC were not only detected in midgut and reproductive organs but also in lower levels in the salivary glands, Malpighian tubules and accessory glands (Fig 4). Analysis of the lifestage profiles indicates that As51 is the most abundantly expressed transcript, as it was detected in high levels throughout all lifestages (Fig 5), and it was the only transcript detected in eggs.

\subsection{In vivo RNAi and subsequent phenotype analysis}

The five groups (consisting of 80 freshly molted $R$. microplus females each) were successfully injected, with $9 \%$ overall mortality. Tick weight after engorgement, mortality rate after placement, egg mass and oviposition efficiency (the percentage of fully fed female (ffF) weight converted into egg mass weight) were monitored for each group. 
Group B and C displayed a significant phenotype, with decreased oviposited egg masses and oviposition efficiencies compared with the control group $(P<0.01)$. The hatching rates of all four dsRNA-injected groups were monitored and compared to the mock-injected group but, no significant change was detected.

\subsection{Silencing confirmation and differential transcriptional interaction analysis}

To confirm that observed phenotypes were in fact due to gene-specific silencing, the percentage silencing of each transcript within its respective dsRNA-injected group was determined, using semi-qPCRs. Expression levels of each transcript were only determined in tissues previously identified to express the transcript in question (Table 2).

The normalized transcript levels of the reprolysin-like transcripts (BmMP1, BmMP2, BmMP4 and BmMP5) (Table 3) were significantly reduced, in the salivary glands of the respective groups $(\mathrm{P}<0.01)$. For BmMP4 and BmMP5 the normalized transcript levels were reduced with $77.15 \%$ and $97.42 \%$, respectively, in the combined BmMP4/BmMP5/dsRNA-injected group (Group A). The normalized levels of BmMP1 and BmMP2 were reduced with $64.54 \%$ and $97.36 \%$, respectively, in the combined BmMP1/BmMP2/BmMP3-dsRNA-injected group (Group B). The normalized transcript level of As51 was significantly reduced, in both the midgut with $55.82 \%(P<0.01)$, the ovaries with $80.42 \%(P<0.01)$ and the salivary glands with $83.75 \%(P<0.01)$ in the combined As51/As70-dsRNA-injected group (Group C), whereas AsC was only significantly silenced within the ovaries with $62.55 \%(P<0.01)$ of the AsC-dsRNA-injected group (Group D), compared to the normalized levels in the mock-injected group (Table 3). Integrated real-time PCR reactions and data analysis revealed that significant non-specific 
silencing took place between As51 and AsC as the normalized level of As51 was reduced with $77.79 \%$ in the midgut and $68.87 \%$ in the ovaries of Group D (Table 3).

The astacin and reprolysin transcripts were silenced in family specific groups, to allow the identification of potential networking on transcriptional level between the five reprolysin, the three astacins, and between two metzincin families, respectively. For this, intergrated semi-qPCR studies were performed, in which the expression level of each transcript was determined within all relevant tissue types in each of the dsRNA-injected groups.

Significant up-regulation of non-silenced transcripts occurred, when one/more of the other metzincin transcript(s) were silenced (Table 3). With the silencing of the two salivary gland reprolysins, BmMP4 and BmMP5 (Group A), the normalized levels of the other salivary gland reprolysins, BmMP1 and BmMP2 significantly increased with $185.32 \%$ and $240.52 \%$, $(P<0.01)$, respectively. Of great interest was that the normalized level of As51 was also significantly increased with $125.44 \%$ in the midgut and $249.95 \%$ in the ovaries $(P<0.01)$. The same effect was observed when the other two salivary gland reprolysins, BmMP1 and BmMP2 (Group B), were silenced (Table 3). The normalized level of BmMP5 was significantly increased with $301.70 \%(P<0.01)$ in the salivary glands and the normalized level As51 with $188.13 \%$ in the midgut and $67.74 \%$ in the ovaries $(P<0.01)$. Upon silencing ofAs51 (Group C) and AsC (Group D), a cross organ response was observed. In this case, the normalized levels of both BmMP1 and BmMP2 were significantly increased in the salivary glands of Group C with $111.44 \%$ and $191.82 \%$ $(P<0.01)$ and with $257.94 \%$ and $181.60 \%$ in Group D. 


\section{Discussion}

By hydrolyzing substrates such as fibrin and fibrinogen, reprolysin metzincins are in effect capable of preventing blood clot formation and dissolving existing clots, during haematophagous feeding, by disaggregating the platelets and fibrin crosslinked matrix (Francischetti et al., 2003). It has also been speculated that such metzincins may play a role in pathogen transmission, since it has been proven that Borrelia spirochetes up regulate the release and activation of matrix metalloprotease gelatinase B (MMP-9) and collagenase 1 (MMP-1) in human cells, improving the transmission of Borrelia burgdorferi across extracellular matrix components (Gebbia et al., 2001). Spirochete dissemination through vertebrate tissues may similarly be aided by salivary metalloproteases and other salivary anti-haemostatic agents (Maritz-Olivier et al., 2007; Ribeiro, 1989), which prevent fibrin clot formation and dissolution of the fibrin clots eventually formed around the feeding cavity of ixodid ticks (Francischetti et al., 2003). For this reason both Decrem et al. (2007) and Imamura et al. (2009) selected salivary gland metalloproteases from $I$. ricinus and $H$. longicornis, respectively, as novel vaccine targets. The results of both studies indicated that vaccination against these putative reproplysin salivary gland enzymes can affect the reproductive fitness of ticks, since the female egg laying abilities were significantly affected.

Another metzincin family of interest is the astacins. Through protein degradation, proteinprotein interaction modulation, ECM turn-over, extracellular coat degradation (hatching) and highly specific cleavage events such as growth factor activation, these enzymes play essential roles in diverse range of physiological mechanisms including digestion (Stöcker 
et al., 1993), early embryo development (dorso-ventral patterning) (Shimell et al., 1991), processing of the ECM (Sterchi et al, 2008) and egg hatching (Young et al., 2000).

This study set out to identify reprolysin and astacin metzincin homologues in $R$. microplus and to evaluate these enzymes as possible anti-R. microplus vaccine targets. Five coding sequences with significant similarity to a known reprolysin-like ixodid tick MP, and three EST sequences with significant similarity to prototype astacin were identified. As with all members of the metzincins (Gomis-Rüth, 2009) the overall similarity and identity among the reprolysins and astacins were low. However, analysis of the amino acid sequences (Figs 1 and 2) confirmed that the sequences contained their respective family's conserved zinc-binding his-motif as well as the conserved methionine, which clearly classified all eight $R$. microplus transcripts as metzincins. Phylogenetic analysis supports the alignment data and indicated that the five reprolysin and three astacin $R$. microplus homologues belong to the reprolysin and astacin metzincin families, respectively (Fig 3).

Expression profiling results indicated that the reprolysins were most abundantly expressed in the salivary glands (Fig 4), whereas the expression of astacins was most abundant in the midgut and reproductive organs (Fig 4). The theoretical signal peptide, GPI-anchor and transmembrane $\alpha$-helix predictions suggest all five $R$. microplus reprolysins to be secreted proteins. By integrating all data it can be postulated that the five reprolysin-like MPs are secreted by the salivary glands into the saliva. Via the saliva these MPs are released into the feeding cavity, where they most probably act on ECM components, to assist in maintaining a fluid intact feeding pool (Francischetti et al., 2003). For the astacinlike MPs it can be speculated, that once a blood meal is obtained, the specialized secretory cells in the midgut epithelium release these enzymes. In the midgut lumen these 
astacin-like MP most probably act on imbibed ECM components. Since both As51 and AsC were detected at high levels in the reproductive organs we hypothesize that these metzincins may also be involved in the reproductive processes, oogenesis and spermatogenesis. As51 is the most abundantly expressed transcript, throughout all lifestages and is the only transcript detected in eggs. Since astacins in other invertebrates are involved in embryogenesis or egg hatching (Bond and Beynon, 1995), it can be hypothesized that this $R$. microplus astacin may have similar function. However, the exact functions and substrates of these $R$. microplus metzincin MPs remain to be determined using native- or recombinant proteins in future studies.

This work presents, to our knowledge, the first description of astacin metalloproteases in ticks and metzincin metalloproteases in tissues other than the salivary glands. Based on the data of this study and other invertebrate studies, it is evident that tick metzincins are not necessarily only involved in ECM digestion but may also be involved in other processes such as oogenesis, spermatogenesis and egg hatching.

To evaluate the vital impact of the eight $R$. microplus metzincins homologues during blood feeding and reproduction, in vivo RNAi was utilized. From the data (Table 2) it was evident that the silencing of the reprolysin-like transcripts BmMP1, 2 and 3 (Group B) and the silencing of the astacin-like transcripts As51 and As70 (Group C) yielded the most significant phenotypes, regarding average egg weight and oviposition efficiency. Normalized transcript levels of the targeted genes (BmMP1, 2, 4, 5 and As51, AsC) were reduced by RNAi some $55-98 \%$ in the respective tissues. Similar degrees of silencing have been reported previously in $R$. microplus (Nijhof et al., 2007). Further, real-time PCR studies and data analysis revealed that significant non-specific silencing took place 
between As51 and AsC: injection of AsC dsRNA, in Group D, not only significantly reduced the normalized levels of $\mathrm{AsC}$, but also resulted in a significant reduction of As51 (Table 3). Therefore it can be reasoned that the non-specific silencing of As51 in Group D also contributed to the observed phenotype of Group D. Although not found to be statistical significant, a possible vice versa effect cannot be excluded. Upon analysis of the alignment of the two transcripts' nucleotide sequences it is evident that there are several conserved regions (as large as 21 consecutive nucleotides), that could have attributed to the non-specific silencing.

The mechanism that impaired the ticks to successfully produce ample amounts of eggs is unknown. However, it is known that it is mandatory for adult female ixodid ticks to have successful blood meals to produce eggs (Soneshine, 1991). With the completion of feeding and digestion a hormonal signal triggers vitellogenesis, a vital process in oogenesis, which produces the yolk nutrient for the developing larvae (Soneshine, 1991). Therefore, it can be hypothesized that silencing of the reprolysin and astacin metalloproteases (within the salivary glands and the midgut) could have indirectly impaired oogenesis by its effect on blood feeding and digestion (Lu et al., 2005; Jia et al., 1996; Sterchi et al., 2008). Since the astacin transcripts are also present in the ovaries and are known to be involved in vital functions such as growth factor activation (Stöcker et al., 1993) and embryo development (dorso-ventral patterning) (Shimell et al., 1991), thus a direct effect on oogenesis cannot be excluded.

The use of integrated semi-qPCRs in this RNAi study also provided novel insight regarding gene regulation between the different members of the metzincin clan investigated. It was found that several fold up-regulation of non-silenced transcripts 
occurred when other transcripts were specifically silenced (Table 3). The remarkable finding was that this was even a cross different organs (Fig 6). Upon silencing of salivary gland reprolysins the midgut and ovary astacins were activated and vice versa. To fully explain these phenomena more integrated transcriptome studies are required, which will provide insight into $R$. microplus metzincin gene regulation during feeding and digestion.

Finally, it can be concluded that by means of a systematic approach we were able to identify five reprolysin and three astacin $R$. microplus homologous and to evaluate the overall vital impact of these metzincins in $R$. microplus blood feeding and reproduction. Due to the significant phenotypes raised, current studies entail BmMP1, BmMP2 and As51 expression to evaluate them as anti- $R$. microplus protective antigens in cattle vaccination trials. As previously mentioned, vaccination with single metzincins did in fact result in some decrease of engorgement weight and ovipositioning, however, it did not have a great effect on tick mortality (Decrem et al., 2007; Imamura et al, 2009). Based on the differential transcriptional network observed between reprolysins and astacins, the results support use of a combinatorial metzincin-based vaccine, which will be evaluated in a subsequent study.

\section{Acknowledgements}

Research on anti-tick vaccines is supported by the Wellcome Trust under the 'Animal Health in the Developing World' initiative through project 075799 entitled 'Adapting recombinant anti-tick vaccines to livestock in Africa'. International research was funded by the University of Pretoria's 'Postgraduate study abroad bursary program'. 


\section{References}

Baffi, M. A., de Souza, G.R.L., Vieira, C.U., de Sousa, C.S., Goulart, L.R.and Bonetti, A.M. (2007). Identification of point mutations in aputative carboxylesterase and their association with acaricide resistance in Rhipichephalus (Boophilus) microplus (Acari: Ixodidae). Vet. Parasitol. 148: 301-309.

Barrett, A. J., Rawlings, N. D., and Woessner, J. F. (2004). Handbook of Proteolytic Enzymes. (ed. Barrett, A. J., Rawlings, N. D., and Woessner, J. F) volume 2, Amsterdam: Elsevier Academic Press., The Netherlands.

Bode, W., Gomis-Rüth, F., and Stöckler, W. (1993). Astacins, serralysins, snake venom and matrix metalloproteinases exhibit identical zinc-binding environments (HEXXHXXGXXH and Met-turn) and topologies and should be grouped into a common family, the 'metzincins'. FEBS Lett. 331: 134-140.

Bond, J. S., and Beynon, R.J. (1995). The astacin family of metalloendopeptidases. Protein Sci. 4: 1247-1261.

Bowles, V.M., Young, A.R., and Barker, S.C. (2007). Metalloproteases and egg-hatching in Pediculus humanus, the body (clothes) louse of humans (Phthiraptera: Insecta). Parasitology 135: 125-130.

Chomczynski, P. (1993). A reagent for the single-step simultaneous isolation of RNA, DNA and proteins from cell and tissue samples. Biotechniques 15: 532-537.

De la Fuente, J., Almazán, C., Canales, M., de la Lastra, J.M.P, Kocan, K. M. and P.Willadsen (2007). A ten-year review of commercial vaccine performance for control of tick infestations on cattle." Anim. Health Res. Rev. 8: 23-28.

De la Fuente, J., Almazán, C., Naranjo, V., Blouin, E.F., and Kocan, K.M. (2006c). Synergistic effect of silencing the expression of tick protective antigens 4D8 and Rs86 in Rhipicephalus sanguineus by RNA interference. Parasitol. Res. 99: 108-113.

De la Fuente, J., Rodríguez, M., Redondo, M., Montero, C., García-García, J.C., Méndez, L., Serrano, E., Valdés, M., Enríquez, A., Canales, M., Ramos, E., de Armas, C.A., Rey, S., Rodríguez, J.L., Artiles, M., and García, L. (1998). Field studies and cost-effectiveness analysis of vaccination with Gavac ${ }^{\mathrm{TM}}$ against the cattle tick Boophilus microplus. Vaccine 16: 366-373.

Decrem, Y., Beaufays, J., Blasioli, V., Lahaye, K., Brossard, M., Vanhamme, L., and Godfroid, E. (2008). A family of putative metalloproteases in the salivary glands of the tick Ixodes ricinus. FEBS J. 275: 1485-1499.

Decrem, Y., Mariller, M., Lahaye, K.,Blasioli, V., Beaufays, J., Boudjeltia, K. Z., Vanhaeverbeek, M., Cérutti, M., Vanhamme, L., and Godfroid, E. (2007). The impact of gene knock-down and vaccination against salivary metalloproteases on blood feeding and egg laying by Ixodes ricinus. Int. J. Parasitol. 38: 549-560 
Dzik, J. M. (2005). Molecules released by helminth parasites involved in host colonization. Acata Biochim Pol. 53: 3364.

Estrada-Pena, A., Bouattour, A., Camicas, J.L., Gulielmone, A., Horak, I., Jongejan, F., Latif, A., Pegram, R., Walker, A.R. (2006). The known distribution and ecological preference of the tick subgenus Boophilus (Acari: Ixodidae) in Africa and Latin America. Exp. Appl. Acarol. 37: 653-662.

Francischetti, I.M.B., Mather, T.N., and Ribeiro, J.M.C. (2003). Cloning of a salivary gland metalloprotease and characterization of gelatinase and fibrin(ogen)lytic activities in the saliva of the Lyme disease tick vector Ixodes scapularis. Biochem. Biophys. Res. Commun. 305: 869-875.

Freije, J.R., and Bischoff, R. (2006). Matrix metallo-proteases. Drug Discov. Today: Technol. 3: 5-11.

Gallego, S.G., Loukas, A., Slade, R.W., Neva, F.A., Varatharajalu, R., Nutman, T.B., Brindley P.J. (2005). Identification of astacin-like metallo-proteinase transcript from the infective larvae of Strongyloides stercoralis. Parasitol. Int. 54: 123-133.

Gebbia, J.A., Coleman, J.L., and Benach, J.L. (2001). Borrelia spirochetes upregulate release and activation of matrix metalloproteinase gelatinase B (MMP-9) and collagense 1(MMP-1) in human cells. Infect. Immunol. 69: $456-462$.

Gomis-Rüth, F.X. (2003). Structural Aspects of the Metzincin Clan of Metalloendopeptidases. Mol. Biotechnol. 24: 157-202.

Gomis-Rüth, F.X. (2009). Catalytic domain architecture of Metzincin metalloproteases. J. Biol. Chem. 284: 1535315357.

Harnnoi, T., Sakahuchi T., Nishikawa, Y., Xuan, X., Fujisaki, K. (2007). Molecular characterization and comparative study of 6 salivary gland metalloproteases from the hard tick, Haemaphysalis longicornis. Comp. Biochem. Physiol. B. 147: 93-101.

Hellemans, J., Mortier, G., De Paepe, A., Speleman, F., and Vandesompele (2007). qBase relative quantification framework and software for management and automated analysis of real-time quantitative PCR data. Genome Biol. 8: R19:11-14.

Iba, K., Albrechtsen, R., Gilpin, B.J., Loechel, F., and Wewer, U.M. (1999). Cysteine-rich domain of human ADAM 12 (meltrin alpha) supports tumor cell adhesion. Am. J. Pathol. 154: 1489-1501.

Imamura, S., da Silva Vaz Junior, I., Konnai, S., Yamada, S., Nakajima, C., Onuma, M. and Ohashi, K. (2009). Effect of vaccination with a recombinant metalloprotease from Haemaphysalis longicornis. Exp. Appl. Acarol. 48: $345-358$. 
Jia, L.G., Shimokawa, K., Bjarnason, J.B., and Fox, J.W. (1996). Snake venom metalloproteinase: structure, function and relationship to the ADAMSs family of proteins. Toxicon 24: 1269-1276.

Jongejan, F., and Uilenberg, G., (2004). The global importance of ticks. Parasitology 129: S3-S14.

Jongejan, F., Nene, V., de la Fuente, J., Pain, A., Willadsen, P. (2007). Advances in the genomics of ticks and tickborne pathogens. Trends Parasitol. 23: 391-396.

Lu, X., Lu, D., Scully, M.F. and Kakkar. V.V. (2005). Snake Venom Metalloproteinase Containing a Disintegrin-like Domain, its Structure-activity Relationships at Interacting with Integrins. Current Medicinal Chemistry: Cardiovascular \& Hematological Agents 3: 249-260.

Macleod, J.A.M., (1978). Ecological studies on ixodid ticks (Acari: Ixodidae) in Zambia. IV. Some anomalous infestation patterns in the northen and eastern regions. Bull Entomol. Res. 68: 409-429.

Maritz-Olivier, C., Stutzer, C., Jongejan, F., Neitz, A.W.H., and Gaspar, A.R.M. (2007). Tick anti-hemostatics: Targets for future vaccines and therapeutics. Trends Parasitol 23(9): 397-407.

Mason, C.A., and Norval, R.A.I, (1980). The ticks of Zimbabwe. 1. The genus Boophilus. Zimbabwe Vet. J. 11: 36-43.

Nijhof, A.M., Balk, J.A., Postigo, M., Rhebergen, A.M., Taoufik, A., Jongejan, F. (2010). Bm86 homologues and novel ATAQ proteins with multiple EGF-like domains from hard and soft ticks. Int. J. Parasitol. 40: 1587-1597.

Nijhof, A.M., Balk, J.A., Postigo, M., Jongejan, F (2009). Selection of refernce genes for quantitative RT-PCR studies in Rhipicephalus (Boophilus) microplus and Rhipicephalus appendiculatus ticks and determination of the expression profile of Bm86. BMC Mol. Biol. 10: 112

Nijhof, A. M., Taoufik, A., de la Fuente, J., Kocan, K. M., de Vries, E. and Jongejan, F. (2007). Gene silencing of the tick protective antigens, Bm86, Bm91 and subolesin, in the one-host tick Boophilus microplus by RNA interference. Int. J. Parasitol. 37: 653-662.

Ribeiro, J.M.C. (1989). Role of saliva in tick/host associations. Exp. Appl. Acarol. 7: 15-20.

Shimell, M.J., Ferguson, E.L., Childs, S.R., and O’Connor, M.B., (1991). The Drosophila dorsal-ventral patterning gene tolloid is related to human bone morphogenetic protein 1. Cell 67(3): 469-481.

Sonenshine, D.E. (1991) Biology of Ticks, (ed. Sonenshine, D.E.) volume 1, New York: Oxford University Press Inc., USA.

Sterchi, E.E., Stöcker, W., and Bond, J.S. (2008 ). Meprins, membrane-bound and secreted astacin metalloproteinases. Mol. Aspects Med. 29: 309-328. 
Stöcker, W., Gomis-Rüth, X.F., Bode, W., and Zwilling, R. (1993). Implications of the three-dimensional structure of astacin for the structure and function of the astacin family of zinc-endopeptidases. Eur. J. Biochem. 214: $215-231$

Tamura, K., Dudley, J., Nei, M., and Kumar, S. (2007). MEGA4: Molecular Evolutionary Genetics Analysis (MEGA) software version 4.0. Mol Biol Evol. 24: 1596-1599.

Thompson, J. D., Gibson, T.J., Plewniak, F., Jeanmougin, F. and Higgins, D.G. (1997). The ClustalX windows interface: flexible strategies for multiple sequence alignment aided by quality analysis tools. Nucleic Acids Res. 24:4876-4882.

Tonnesen, M.H., Penzhorn, B.L., Bryson, N.R., Stoltsz, W.H., and Masibigiri, T. (2004). Displacement of Boophilus decoloratus by Boophilus microplus in the Soutpansberg region, Limpopo Province, South Africa. Exp. Appl. Acarol. 32: 199-209.

Willadsen, P. (2004). Anti-tick vaccines. Parasitology 129: S367-S387.

Williamson, A.L., Lustigman, S., Oksov, Y., Deumic, V., Plieskatt, J., and Mendez, S., (2006). "Ancylostoma caninum MTP-1, an astacin-like metalloprotease secreted by infective hookworm larvae, is involved in tissue migration. Infect. Immun. 74: 961-967.

Young, A.R., Mancuso, N., Meeusen, E.N.T., and Bowels, V.M. (2000). Characterisation of proteases invovled in egg hatching of the sheep blowfly, Lucilia cuprina. Int. J. Parasitol. 30: 925-932. 
Table 1. Characteristics of oligonucleotide primers used.

\begin{tabular}{|c|c|c|c|c|}
\hline $\begin{array}{l}\text { Target } \\
\text { name }\end{array}$ & $\begin{array}{c}\text { Primer } \\
\text { name }\end{array}$ & Primer seq 5'- 3' & $\begin{array}{c}\begin{array}{c}\text { Product } \\
\text { size }\end{array} \\
\end{array}$ & Purpose \\
\hline BmMP1 & tpBmMP1fw & GCA AGA GAG ATC AAC CGA AAG & & Expression profiling \\
\hline BmMP1 & tpBmMP1rv & AAA GAG AAG TTT GTC CGC AAG TA & 100 & Expression profiling \\
\hline BmMP2 & tpBmMP2fw & AAT CCG TGC GCT ATT GTT GCT AC & & Expression profiling \\
\hline BmMP2 & tpBmMP2rv & GCC AAT CCA TCG TGA ATG CTA AC & 132 & Expression profiling \\
\hline BmMP3 & tpBmMP3fw & TAA GTG AAG ACA TAA CGC TGA AC & & Expression profiling \\
\hline BmMP3 & tpBmMP3rv & GCT CGT GGT CGT CGT AAA & 144 & Expression profiling \\
\hline BmMP4 & tpBmMP4fw & CGG GAC ACA CTT ACG ACG GAA TA & & Expression profiling \\
\hline BmMP4 & tpBmMP4rv & TCC ATG TCA GCG GTC ACG TT & 103 & Expression profiling \\
\hline BmMP5 & tpBmMP5fw & ACG GAA CGA AAT GAC ACA TAT CA & & Expression profiling \\
\hline BmMP5 & tpBmMP5rv & GCA GAT CCA ACA AAG GCA TAA C & 128 & Expression profiling \\
\hline As51 & tpAs5051fw & AAC CGA CTG CTG ACG CCA TT & & Expression profiling \\
\hline As51 & tpAs5051rv & CTC TGT CAA GTG ACT GCC GTC CT & 117 & Expression profiling \\
\hline AsC & tpAsCfw & TTG AGG TGA CTG CCA TTC TTC GC & & Expression profiling \\
\hline AsC & tpAsCrv & AGA ACA GGT TGC TGA CGC CCT TC & 115 & Expression profiling \\
\hline As70 & As70Nqfw & TTG GTG TGG TGT AAG TTG ACC CT & & Expression profiling \\
\hline As70 & As70Nqrv & GAA GTG ATT TGC CTG CCG TTT A & 116 & Expression profiling \\
\hline BmMP1 & iBmMP1fw & TAA TAC GAC TCA CTA TAG GGA GAT ACC AGA AGG CGA GAG GTC AAT G & & dsRNA synthesis \\
\hline BmMP1 & iBmMP1rv & TAA TAC GAC TCA CTA TAG GGA GAA CAA GAA GCA GGG CAT CGT G & 487 & dsRNA synthesis \\
\hline BmMP2 & iBmMP2fw & TAA TAC GAC TCA CTA TAG GGA GAG CAC GCT TGT GGT TCA CGA A & & dsRNA synthesis \\
\hline BmMP2 & iBmMP2rv & TAA TAC GAC TCA CTA TAG GGA GAC GCA TCA GGA TCG CCA TAT AGA T & 635 & dsRNA synthesis \\
\hline BmMP3 & iBmMP3fw & TAA TAC GAC TCA CTA TAG GGA GAT AAG TGA AGA CAT AAC GCT GAA C & & dsRNA synthesis \\
\hline BmMP3 & iBmMP3rv & TAA TAC GAC TCA CTA TAG GGA GAG CTC GTG GTC GTC GTA AA & 144 & dsRNA synthesis \\
\hline BmMP4 & iBmMP4fw & TAA TAC GAC TCA CTA TAG GGA GAC CAC GCC ACA GGA TTG AAC & & dsRNA synthesis \\
\hline BmMP4 & iBmMP4rv & TAA TAC GAC TCA CTA TAG GGA GAT ATC GTT GCC ATT TCT GTA AGG G & 379 & dsRNA synthesis \\
\hline BmMP5 & iBmMP5fw & TAA TAC GAC TCA CTA TAG GGA GAA TTG CGG AAC ATC ATT ACC ATA A & & dsRNA synthesis \\
\hline BmMP5 & iBmMP5rv & TAA TAC GAC TCA CTA TAG GGA GAG GTT TCT AAG GGC ACT GTA TCG T & 451 & dsRNA synthesis \\
\hline As51 & iAs5051fw & TAA TAC GAC TCA CTA TAG GCG TGA CGG AGC CGA ATA GAC TGT & & dsRNA synthesis \\
\hline As51 & iAs5051rv & TAA TAC GAC TCA CTA TAG GAA TGG CGT CAG CAG TCG GTT C & 610 & dsRNA synthesis \\
\hline As70 & iAs8770fw & TAA TAC GAC TCA CTA TAG GAC CTG CCT GCG ATT TGT TGA & & dsRNA synthesis \\
\hline As70 & iAs8770rv & TAA TAC GAC TCA CTA TAG GCG CAT CCG CAG AAT ACA GCA T & 315 & dsRNA synthesis \\
\hline AsC & iAsContigfw & TAA TAC GAC TCA CTA TAG GGA GAG TGT TTT CTG GCT CCA CGT T & & dsRNA synthesis \\
\hline AsC & iAsContigrv & TAA TAC GAC TCA CTA TAG GGA GAA TGC GGT TAC TAA GAA GGA CGA G & 367 & dsRNA synthesis \\
\hline BmMP1 & qBmMP1fw & GCA AGA GAG ATC AAC CGA AAG & & Real-time PCR \\
\hline BmMP1 & qBmMP1rv & AAA GAG AAG TTT GTC CGC AAG TA & 100 & Real-time PCR \\
\hline BmMP2 & qBmMP2fw & AAT CCG TGC GCT ATT GTT GCT AC & & Real-time PCR \\
\hline BmMP2 & qBmMP2rv & GCC AAT CCA TCG TGA ATG CTA AC & 132 & Real-time PCR \\
\hline BmMP3 & qBmMP3fw & TTA TGA CAG GAT CGG CTA ATC TA & & Real-time PCR \\
\hline BmMP3 & qBmMP3rv & TTA TAT GAA CCA TGT ACG GCT CT & 120 & Real-time PCR \\
\hline BmMP4 & qBmMP4fw & AAC TGA CGC TGA ACC TCA GAA AG & & Real-time PCR \\
\hline BmMP4 & qBmMP4rv & CCG TTG AAG AAA TGT GTC ACT TC & 101 & Real-time PCR \\
\hline BmMP5 & qBmMP5fw & ACG GAA CGA AAT GAC ACA TAT CA & & Real-time PCR \\
\hline BmMP5 & qBmMP5rv & GCA GAT CCA ACA AAG GCA TAA C & 128 & Real-time PCR \\
\hline As51 & qAs5051fw & AAC CGA CTG CTG ACG CCA TT & & Real-time PCR \\
\hline As51 & qAs5051rv & CTC TGT CAA GTG ACT GCC GTC CT & 117 & Real-time PCR \\
\hline AsC & qAsCfw & TTG AGG TGA CTG CCA TTC TTC GC & & Real-time PCR \\
\hline AsC & qAsCrv & AGA ACA GGT TGC TGA CGC CCT TC & 115 & Real-time PCR \\
\hline As70 & qAs70Nqfw & TTG GTG TGG TGT AAG TTG ACC CT & & Real-time PCR \\
\hline As70 & qAs70Nqrv & GAA GTG ATT TGC CTG CCG TTT A & 116 & Real-time PCR \\
\hline
\end{tabular}


Table 2. Tick engorgement weight, egg mass weight and oviposition efficiency of double-stranded RNA (dsRNA)-injected B. microplus ticks, injected as freshly molted females. (ffF: fully fed females)

\begin{tabular}{|c|c|c|c|c|}
\hline Group & Number of ticks & $\begin{array}{l}\text { Average ffF weight }{ }^{\mathrm{a}} \\
\text { (mg) }\end{array}$ & Average egg weight ${ }^{\mathrm{b}}(\mathrm{mg})$ & $\begin{array}{l}\text { Average ffF to } \text { egg }^{\mathrm{c}} \\
\text { (\%) }\end{array}$ \\
\hline TE & 68 & $328 \pm 49$ & $152 \pm 63$ & $46 \%$ \\
\hline A & 68 & $364 \pm 71$ & $129 \pm 54$ & $37 \%$ \\
\hline B & 74 & $352 \pm 58$ & $93 \pm 56^{*}$ & $27 \%$ ** \\
\hline C & 79 & $341 \pm 73$ & $89 \pm 72^{* *}$ & $26 \%$ ** \\
\hline D & 75 & $313 \pm 63$ & $111 \pm 38$ & $37 \%$ \\
\hline
\end{tabular}

${ }^{a}$ Ticks which completed feeding and those removed 14 days after mock- or dsRNA-injection were weighed, the average $\pm S D$ weight were calculated and compared between dsRNA and mock-injected control ticks, using the Student's t-test with unequal variance.

${ }^{\mathrm{b}}$ The egg mass oviposited by each tick was weighed individually and compared between dsRNA and mockinjected control ticks, using one-way ANOVA and Tukey-Kramer multiple comparison test.

${ }^{c}$ The percentage fully fed female weight converted into egg mass was calculated for each tick individually and compared between dsRNA and mock-injected control ticks, using one-way ANOVA and Tukey-Kramer multiple comparison test.

( $\left.\mathrm{*}<<0.01,{ }^{*} \mathrm{P}<0.001\right)$. 
Table 3. Silencing confirmation and differential transcriptional response analysis. The relative transcript levels in the salivary glands (SG), midgut (MG) and ovaries (O) of five partially fed females, 6 days after injected with dsRNA of BmMP4 and BmMP5 (Group A), BmMP1, BmMP2 and BmMP3 (Group B), As51 and As70 (Group C) and AsC (Group D) are represented by the calibrated normalized relative quantity (CNRQ)values ( \pm SD), normalized against ELF1- $\alpha$ and PPIA. The correlated percentage up (+) or down (-) regulation of each transcript within its dsRNA silenced group (in bold) and in the other groups are compared to its level in the control group.

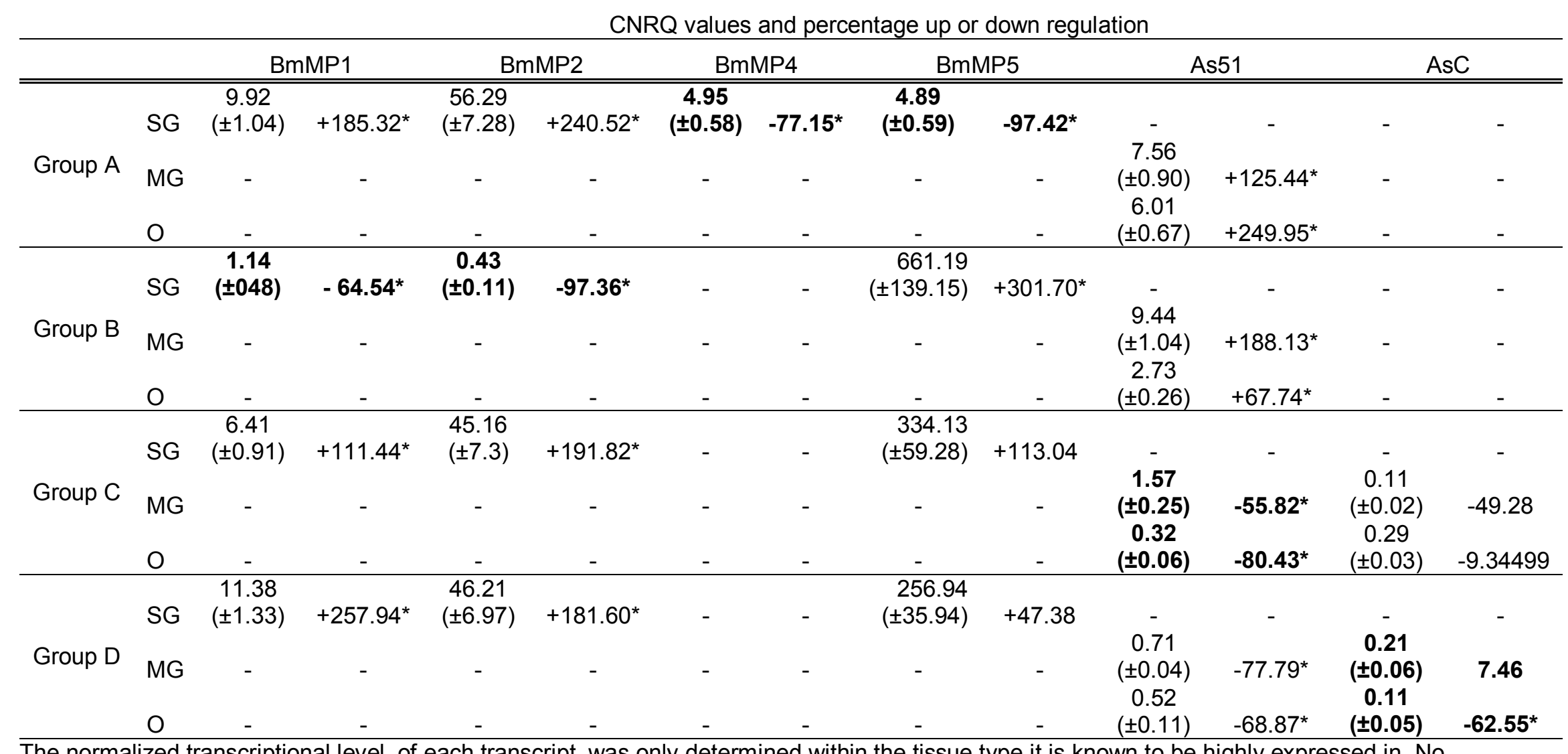

The normalized transcriptional level, of each transcript, was only determined within the tissue type it is known to be highly expressed in. No subsequent real-time analysis was performed for BmMP3 and As70, since both transcripts had very low expression levels. ${ }^{*}$ Denotes if the difference is significant, as determined by the student's test $\left({ }^{*} \mathrm{P}<0.01\right)$. 


\section{Legends of Figures}

Fig 1. The amino acid alignment of the five reprolysin-like $R$. microplus sequences against

scapularis MP1 (IsMP1: GenBank accession no. AAP22067), I. ricinus Metis 1 (GenBank accession no. $\underline{\text { AM747806) }}$ and two reprolysin SVMP, atrolysins. Sequence similarity is represented by grey scaling, with black indicating $100 \%$ similarity, grey $80-90 \%$ similarity and light grey $60-70 \%$ similarity. The conserved catalytic histidine -domain is indicated by the black box and the conserved methionine with a \#. The atrolysin sequences correspond to NCBI accession nos. gi|402259| (Crotalus atrox atrolysin B) and gi|213030| (C. atrox atrolysin E).

Fig 2. Amino acid alignment of the three astacin-like $R$. microplus sequences against prototype atacin (GenBank accession no. CAA64981.1) and astacin-like MP toxin precursor of Loxosceles intermedia (LiMP: GenBank accession no. ABK20019.1). Sequence similarity is represented by grey scaling, with black indicating $100 \%$ similarity, grey $80-90 \%$ similarity and light grey $60-70 \%$ similarity. The conserved catalytic histidine -domain is indicated by the black box and the conserved methionine with a \#.

Fig 3. Phylogenetic analysis of the five reprolysin-like and three astacin-like $R$. microplus MPs together with other ixodid tick MPs and astacin. Ir refers to I. ricinus (Metis 1 (GenBank accession no. $\underline{\mathbf{A M 7 4 7 8 0 6}}$ ) and Metis 2 (GenBank accession no. AM747807), Is refers to I. scapularis (IsMP1: GenBank accession no. AAP22067), Bm refers to R. microplus (BmMP1: gi|71726988, BmMP2 gi|71726992, BmMP3: gi|71726990, BmMP4: gi|71726986, BmMP5: gi|7172684, As51: gi|82845051, As70: gi|49558770 and AsContig: assembled from gi|82852194, gi|82845107 and gi|82843217. Aa refers to A. astacus (astacin: GenBank accession no. CAA64981.1). Boodstrap values are shown on the lineage of the tree.

Fig 4. Expression profiling of reprolysin- and astacin-like metzincins in R. microplus adult tissues. Fig A: Female tissue: lane 1: engorged female haemolymph, lane 2: partially fed female midgut, lane 3: partially fed female malpighian tubules, lane 4: partially fed female ovaries, lane 5: partially fed female salivary glands, lane 6: unfed female midgut, lane 7: unfed female salivary glands, lane 8: partially fed female fatbody, (B: Male tissues) Lane 1: fed male ancillary glands, lane 2: fed male midgut, lane 3: fed male salivary glands, lane 4: 
unfed male ancillary glands, lane 5: unfed male midgut, lane 6: unfed male salivary glands and lane 7: unfed male testis.

Fig 5. Expression profiling of metzincins in B.microplus feeding lifestages. Lane 1: eggs, lane 2: unfed larvae, lane 3: engorged larvae, lane 4: unfed nymphs, lane 5: engorged nymphs, lane 6: unfed female, lane 7: unfed male, lane 8: partially fed female, lane 9: fed male, lane 10: carcass and lane 11: no template control.

Fig 6. Observed transcriptional responses between metzincins of the salivary gland and gut tissues. In the first experiment (green background) midgut astacins were silenced (red arrow) resulting in the up regulation of salivary gland reprolysins (black arrow). In a second experiment (purple background), the observation was supported by up regulation of midgut astins (black arrow) in reprolysin silenced (red arrow) ticks. 


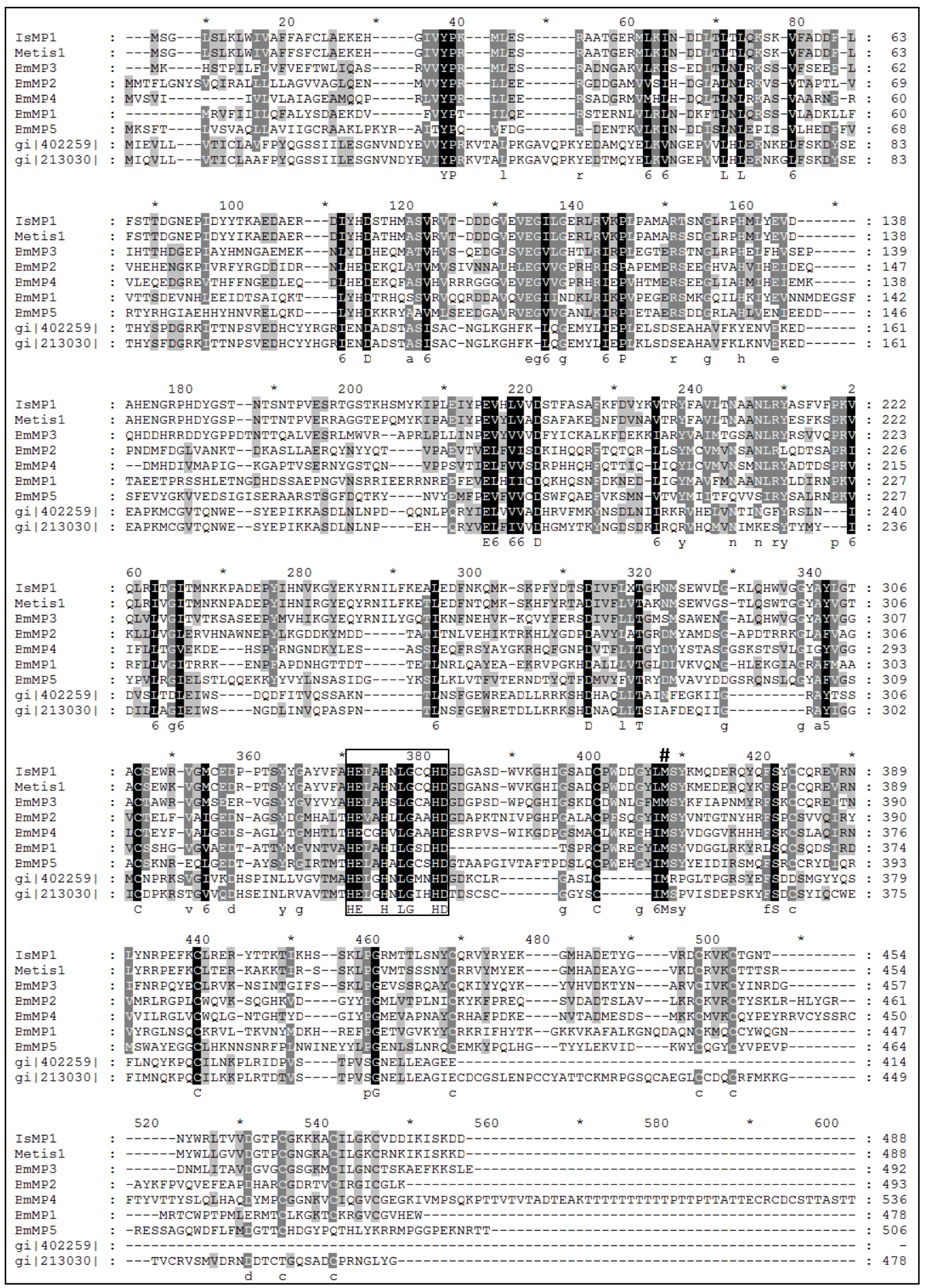

Figure 1 


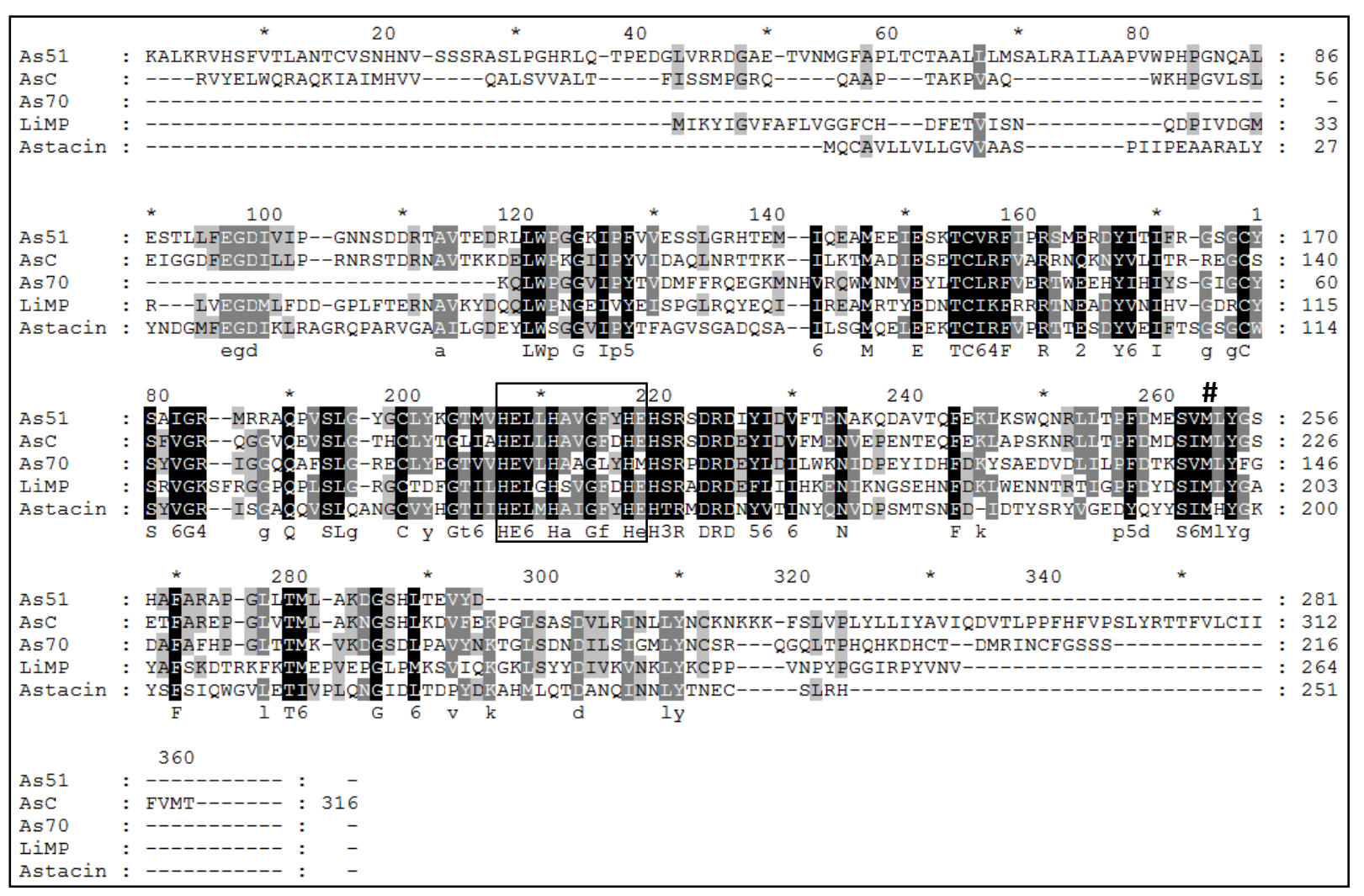

Figure 2 


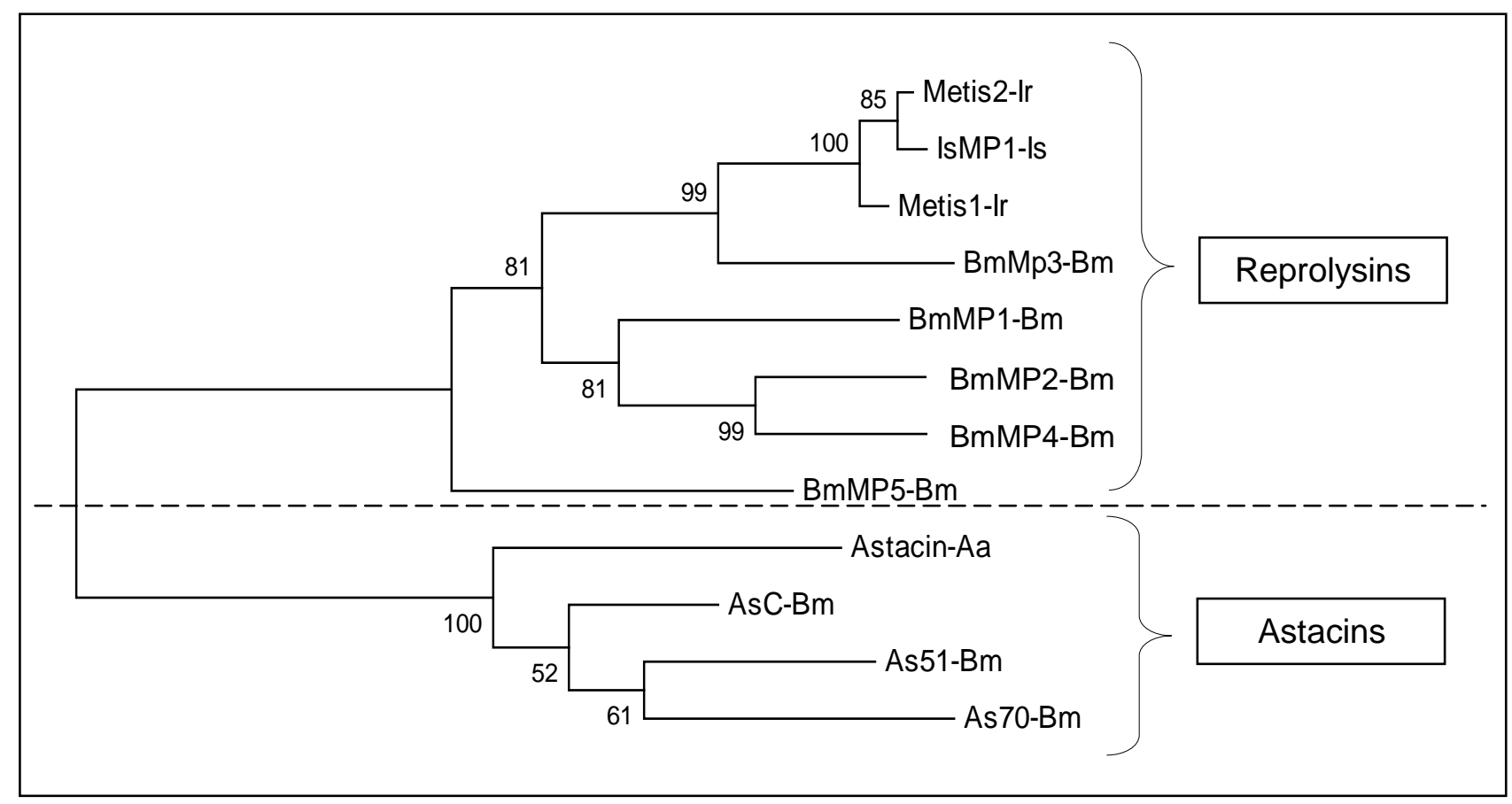

Figure 3 


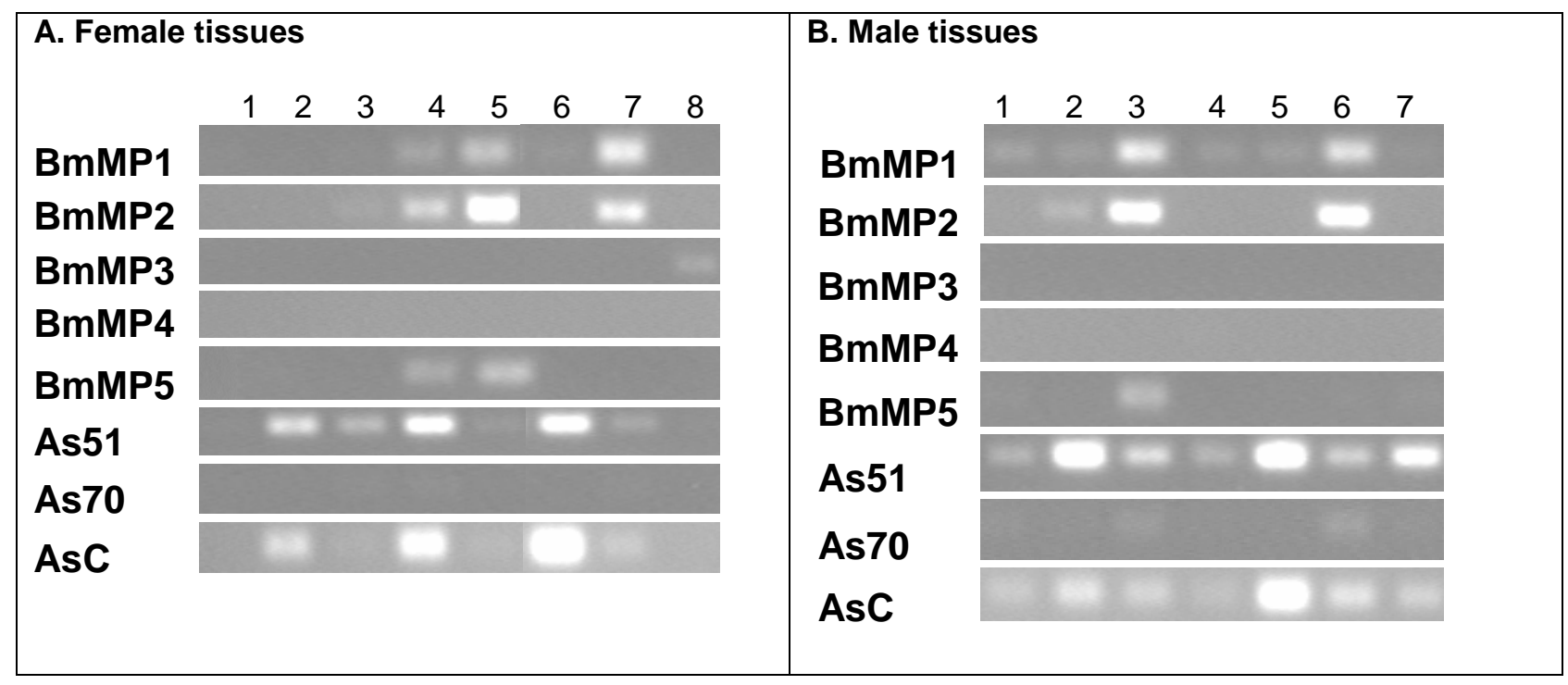

Figure 4. 


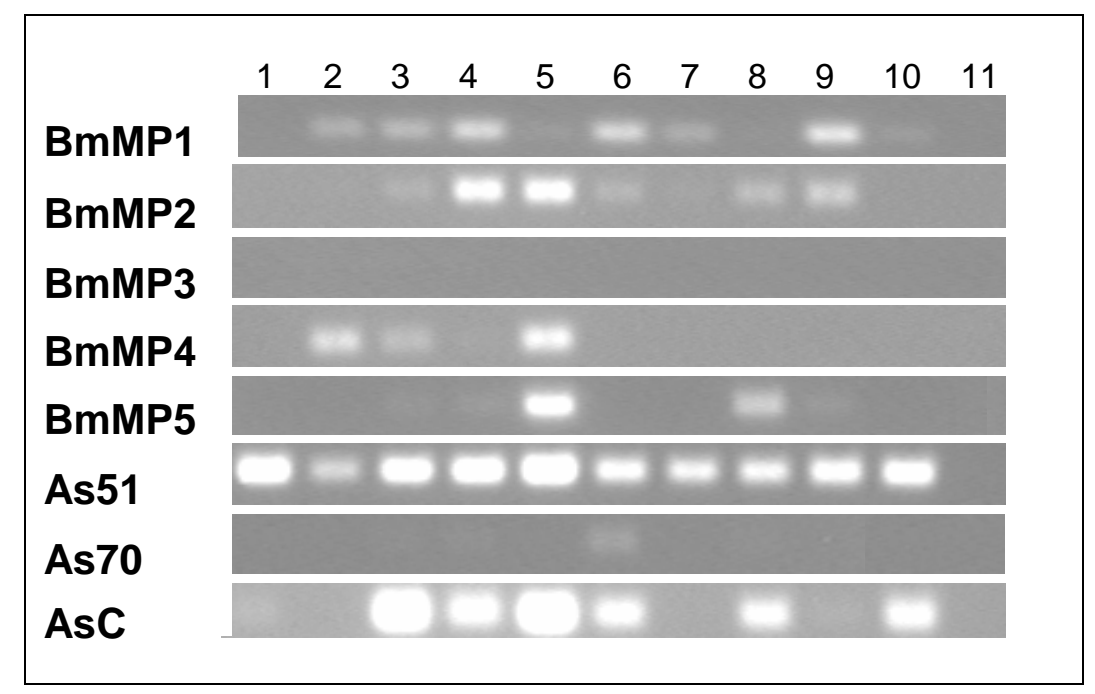

Figure 5. 


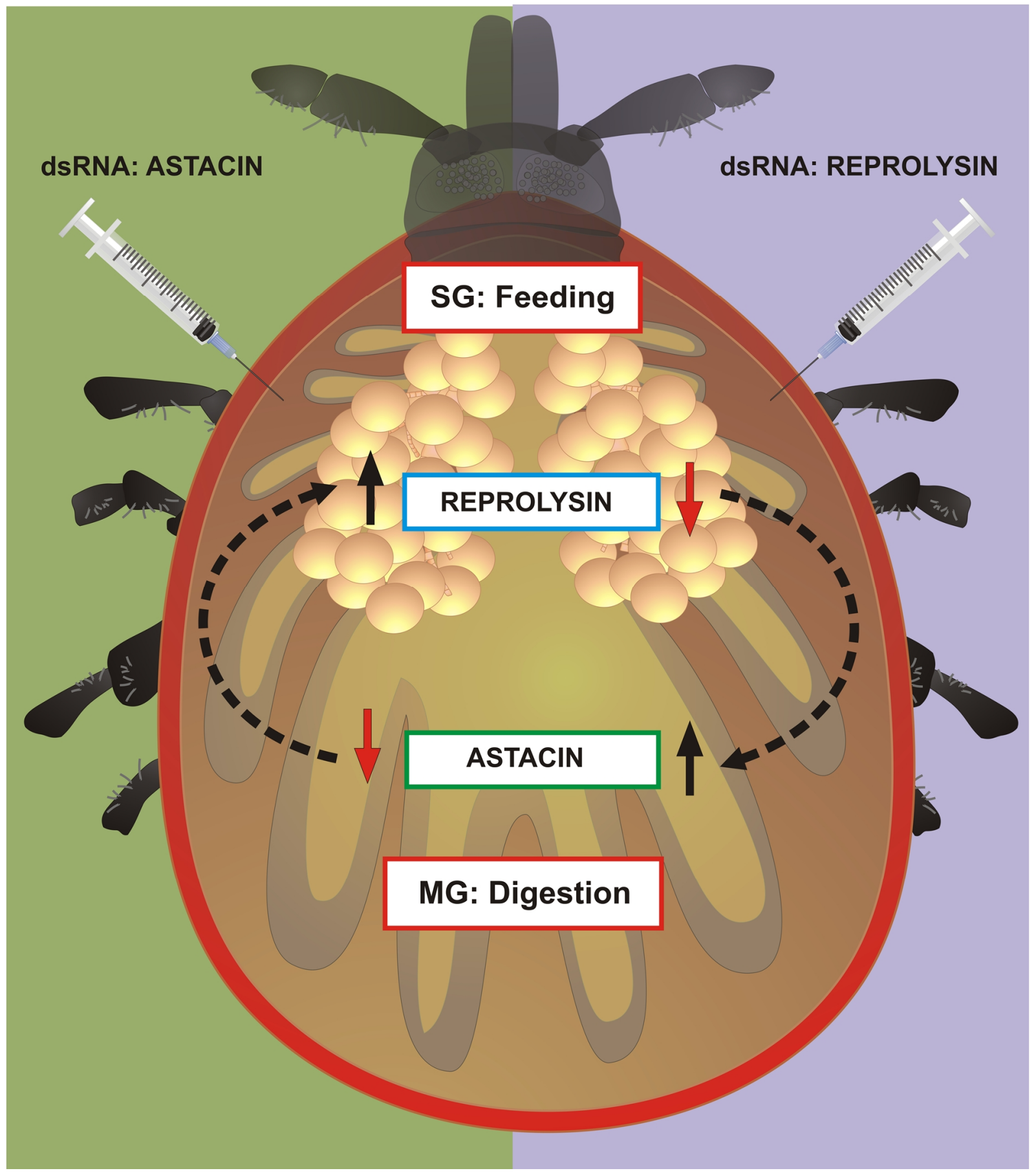

Figure 6. 